\title{
The Benefits and Risks of New Therapies for Type 2 Diabetes
}

\author{
Carl V. Asche, PhD, and Richard E. Nelson, PhD
}

A pproximately $7.8 \%$ of the U.S. population has either diagnosed $(5.9 \%)$ or undiagnosed $(1.9 \%)$ diabetes, and $90 \%-95 \%$ of cases of diabetes in adults are type $2 .{ }^{1}$ Because of the disease and related complications, diabetes places a significant financial burden on the health care system; the total cost of diabetes in the United States in 2007 was an estimated $\$ 174$ billion, of which $\$ 116$ billion were for treatment of the disorder and $\$ 58$ billion were attributable to reduced productivity. ${ }^{2}$ In 2007, 1.6 million people aged 20 or older were newly diagnosed with diabetes in the United States. ${ }^{1}$ Current guidelines issued by the American Diabetes Association (ADA) recommend a combination of lifestyle change and treatment with metformin, if not contraindicated, at the time of diagnosis, and an established treatment goal of attaining and maintaining hemoglobin Alc (Alc) less than $7.0 \% .^{3}$

Recent figures indicate that more patients with type 2 diabetes are able to achieve glycemic control than in the past. In $1999-2000,37.0 \%$ of patients with type 2 diabetes achieved glycemic control as defined by Alc level less than $7 \%$. This number rose to $55.7 \%$ in 2003-2004. ${ }^{4}$ However, although treatment with metformin will initially reduce Alc and result in significant weight reduction, Riedel et al. found that nearly one third of patients will experience "treatment failure," which they defined as at least 1 Alc value of $7 \%$ or greater, on metformin monotherapy with the average time to treatment failure approximately one and one half years. ${ }^{5}$ Thus, it is not surprising that over one third of new type 2 diabetic patients who remain on antidiabetic pharmacotherapy for at least 12 months have at least 1 change in their treatment regimen during that time. ${ }^{6}$ Treatment strategies are intended to delay or prevent failure to maintain desirable Alc in the hope of preventing complications.

As with all new agents, determining place in therapy is important for payers and providers, and in the case of type 2 diabetes, predicting treatment success or failure with initial therapy will factor into immediate and long-term treatment strategies.

In the June 2008 issue of JMCP, VanDeKoppel et al. presented a review of the safety and efficacy of 3 new agents (exenatide, pramlintide and sitagliptin) used to treat type 2 diabetes, as well as an effort to define their place in therapy. ${ }^{7}$ The authors performed their review via a MEDLINE search (1950 to June 2007, including systematic reviews and meta-analyses through December 2007) for English-language articles of studies of human subjects using search terms that included type 2 diabetes and generic names of the 3 drugs. VanDeKoppel et al. reported that all 3 agents have demonstrated a modest effect on reducing Alc, and 2 of the 3 agents have modest positive effect on weight loss; sitagliptin was the agent that has not been associated with weight loss.

VanDeKoppel noted that the long-term safety and efficacy of these agents are unknown and that these agents are more costly than the first-line agents, metformin and sulfonylureas, both available in generic form. The authors concluded that the newer agents should be reserved for patients who are not adequately managed by older agents that have known long-term efficacy and safety and are usually available at much lower cost.

VanDeKoppel et al.'s review puts the value of these 3 agents into context by providing a comprehensive literature review of these new agents and presenting the current (2008) real world prices of all of the drugs in the treatment guidelines to help determine their place in therapy. Overall, VanDeKoppel et al. have made important and valid points addressing the best way to determine the relative value of these 3 new agents.

Patients often do not possess the knowledge necessary to make choices between new and established therapies. Efforts to increase public awareness of the relative value of new drug therapies are often underpowered compared with the influence of drug companies over physicians' and patients' assessments of the benefits and risks associated with new agents. The influence that drug companies have over the perception of their products has markedly increased since the 1997 regulatory shift on the part of the U.S. Food and Drug Administration (FDA) that led to the increasing use of direct-to-consumer (DTC) pharmaceutical advertising in the United States. The introduction and growth of this form of advertising make it evident that the news media might play an important role in offsetting the DTC advertising phenomenon. However, a study by Hartley and Coleman, in which the authors analyzed print news coverage from August 1997 to July 2003, found that the pharmaceutical industry is given more prominence, as measured by the number of times mentioned and the number of times quoted, than are other stakeholders (providers, consumers, corporate purchasers, state, or DTC critics). ${ }^{8}$

VanDeKoppel et al's article in JMCP is neutral and factual in examining the findings from the short-term studies available to date including the reports of adverse effects, such as the potential for severe allergic and dermatologic reactions with sitagliptin. They urge caution on the part of patients and providers because of the lack of long-term effectiveness and safety information for 
these new agents. Although it is true that these new agents have not benefited from the attention that conventional treatments have received, this relative lack of attention has occurred primarily because the new agents have not been marketed long enough for the accumulation of long-term efficacy and safety data. Yet, for each of these agents, VanDeKoppel et al. present encouraging preliminary short-term research information about their efficacy, mechanisms of action, and safety. Specifically, the authors report on the Alc-lowering effects (by $0.5 \%$ to $1 \%$ for exenatide and pramlintide, and by $0.5 \%$ to $0.7 \%$ for sitagliptin). However, the authors also report notable side effects for these agents (nausea in exenatide and pramlintide, severe hypoglycemia in pramlintide, and severe allergic and dermatologic reactions with sitagliptin).

Additional studies with longer time frames for each of these 3 drugs have shown results consistent with those presented by VanDeKoppel et al. For example, in an open-ended, open-label extension of 3 clinical trials, exenatide was shown to reduce Alc (mean $[\mathrm{SE}]-1.1 \%$ [0.1\%] $\mathrm{P}<0.001$ ) from baseline to 12 weeks and maintain this reduction for 3 years in patients on metformin and/or a sulfonylurea; $46 \%$ of patients achieved Alc of $7 \%$ or less. ${ }^{9}$ In the same clinical trials, patients lost weight (-5.3 [0.4] $\mathrm{kg}, P<0.001$ ) over the 3 -year period. Those patients with serum lipid laboratory values experienced decreases in triglycerides (by $12 \%, P<0.001$ ), total cholesterol (by $5 \%, P<0.001$ ), and lowdensity lipoprotein cholesterol (by $6 \%, P<0.001$ ), and increases in high-density lipoprotein cholesterol (by $24 \%, P<0.001$ ) ${ }^{9}$ In two 52-week trials, type 2 diabetes patients on pramlintide experienced Alc reductions of $0.60 \%-0.62 \%$ and body weight reductions of 1.3-1.4 kg. ${ }^{10}$ In a subsequent placebo-controlled study, patients on pramlintide experienced weight loss (mean [SE] - 1.6 [0.3] kg for pramlintide vs. +0.7 [0.3] kg for placebo, $P<0.001$ ) and decreases in high-sensitivity C-reactive protein $(-0.8$ [0.2] $\mathrm{mg}$ per liter for pramlintide vs. 0.1 [0.2] mg per liter for placebo, $P<0.01){ }^{11}$ Individuals at high cardiovascular risk (baseline triglycerides at least $150 \mathrm{mg}$ per $\mathrm{dL}$ ) experienced decreases in triglycerides ( -43 [14] mg per dL $P<0.05$ ). ${ }^{11}$ Finally, although no evidence is available for a cardiovascular benefit for sitagliptin, in a 52-week active-comparator study (Sitagliptin Study 024 Group) in which patients with inadequate glycemic control with metformin were randomized to receive either sitagliptin or glipizide as adjunctive therapy, both drugs reduced Alc by $-0.67 \%$ from a mean baseline of $7.5 \%$, showing that sitagliptin was not inferior to glipizide. ${ }^{12}$ In the Sitagliptin Study 024 Group, patients taking sitagliptin experienced mean weight loss of $-1.5 \mathrm{~kg}$ compared with mean weight gain of $+1.1 \mathrm{~kg}$ for glipizide, yielding a between-group difference of $-2.5 \mathrm{~kg}$ (95\% CI=-3.1 to -2.0 , $P<0.001$ ), and the proportion reporting hypoglycemic events was lower for sitagliptin-treated patients (5\%) than for glipizidetreated patients $(32 \%, P<0.001){ }^{12}$

While glycemic control is clearly the intermediate and primary outcome of these short-term studies, the apparent weight loss and favorable changes in serum lipids suggest possible future value that may be manifest in end point outcomes. While weight loss in diabetic patients has been shown to improve glycemic control, ${ }_{13}^{13}$ it remains to be determined if the modest weight loss observed with exenatide, pramlintide, and perhaps an absence of weight gain with sitagliptin will persist long term and be associated with end point outcomes.

In a recent editorial, Nathan criticized the decision by the FDA to approve sitagliptin, "one of the less effective glycemia-lowering drugs introduced in recent years," despite little evidence of its long-term efficacy and safety. ${ }^{14}$ In his opinion, a major barrier to good diabetes care is the failure of clinicians and their patients to effectively implement currently available interventions, in part because clinicians receive most of their information directly from drug company representatives and promotional material from drug companies. ${ }^{14}$ For this reason, more peer-reviewed studies on the long-term risks and benefits of these newer diabetes medications are crucial in determining their therapeutic niches. A recent (2008) Cochrane Systematic Review of 25 studies of dipeptidyl peptidase-4 (DPP-4) inhibitors, the class of drugs to which sitagliptin belongs, concluded that the DPP-4 inhibitors have "theoretical advantages" over conventional therapies, but longterm data are unavailable to determine whether these advantages truly exist..$^{15}$ Due to their infrequent nature, many severe adverse events are not detected in small, short-term studies, as evidenced recently by reports of several cases of hemorrhagic or necrotizing pancreatitis in patients taking exenatide. ${ }^{16} \mathrm{~A}$ step in the right direction was taken last month when a panel of FDA advisors recommended that, besides lowering blood sugar, all drugs designed to control type 2 diabetes should also be tested through long-term cardiovascular trials. ${ }^{17}$ However, we also advocate the use of real-world, retrospective evaluations of these medications as these studies can potentially include more patients than clinical trials, thus improving the chances of detecting infrequent adverse events. In addition, such studies can compare the use of a variety of drugs in a way that represents actual clinical practice, unlike controlled clinical trials which typically compare 1 study drug to placebo.

The evidence presented by VanDeKoppel et al. is consistent with that presented earlier this year by the ADA and the European Association for the Study of Diabetes (EASD) as the ADA-EASD 2008 Consensus Algorithm..$^{18}$ The ADA-EASD algorithm shows expected Alc reductions in the range of 0.5\%-1.0\% with both exenatide and pramlintide and lists "weight loss" as an "advantage" with both agents, compared with expected Alc reduction in the range of $0.5-0.8 \%$ with sitagliptin and "weight neutral" as an "advantage."

We should incorporate the best evidence available on newly approved medications while continuing to study and monitor these drugs to be able to adequately and accurately place them within the spectrum of diabetes treatment. Diabetes medications that aid in glycemic control while demonstrating beneficial, or at least non-harmful, effects on body weight and biomarkers such 
as serum lipids will potentially be important tools in treating this disease. We do agree, however, that more research is needed to determine the proper therapeutic place for these new medications. With the ADA-EASD 2008 Consensus Algorithm using the word "expensive" to describe each of these new medications, economic analyses are especially important. Information from clinical trials in which the drugs were compared against placebo is valuable in the FDA approval process. But comparative effectiveness and cost-effectiveness studies using real-world data are also important, particularly in diabetes treatment where there are already 9 different classes of medications and plenty of opportunities to compare multiple treatment options.

\section{Authors}

CARL V. ASCHE, PhD, is Research Associate Professor and Director of Graduate Studies, and RICHARD E. NELSON, PhD, is Health Economics and Outcomes Research Fellow, Pharmacotherapy Outcomes Research Center, University of Utah College of Pharmacy, Salt Lake City, UT.

AUTHOR CORRESPONDENCE: Carl V. Asche, PhD, University of Utah College of Pharmacy, 421 Wakara Way, \#208, Salt Lake City, UT 84108. Tel.: 801.587.9715; E-mail: carl.asche@pharm.utah.edu

\section{DISCLOSURES}

The outcomes research center at The Pharmacotherapy Outcomes Research Center receives grants from manufacturers of diabetes drugs. Asche has performed diabetes work for Novartis on a University of Utah grant that was not drug specific and has served on a Novo Nordisk diabetes advisory board. Nelson reports no relationships with manufacturers of diabetes drugs.

\section{REFERENCES}

1. National Diabetes Information Clearinghouse: National Diabetes Statistics, 2007. Available at: www.diabetes.niddk.nih.gov/dm/pubs/statistics/ Accessed August 22, 2008

2. American Diabetes Association. Economic costs of diabetes in the U.S. in 2007. Diabetes Care. 2008;31(3):596-615. Available at: www.care.diabetesjournals.org/misc/econcosts.pdf Accessed August 20, 2008.

3. Standards of medical care in diabetes-2008. (suppl) Diabetes Care. 2008;31 Suppl 1:S12-54. Available at: www.care.diabetesjournals.org/cgi/ reprint/31/Supplement_1/S12 Accessed August 20, 2008.

4. Hoerger TJ, Segal JE, Gregg EW, Saaddine JB. Is glycemic control improving in U.S. adults? Diabetes Care. 2008;31(1):81-86.

5. Riedel AA, Heien H, Wogen J, Plauschinat CA. Loss of glycemic control in patients with type 2 diabetes mellitus who were receiving initial metformin, sulfonylurea, or thiazolidinedione monotherapy. Pharmacotherapy. 2007;27(8):1102-10.

6. Boccuzzi SJ, Wogen J, Fox J, Sung JC, Shah AB, Kim J. Utilization of oral hypoglycemic agents in a drug-insured U.S. population. Diabetes Care. 2001;24(8):1411-15.

7. VanDeKoppel S, Choe HM, Sweet BV. Managed care perspective on three new agents for type 2 diabetes. J Manag Care Pharm. 2008;14(4):363-80. Available at: www.amcp.org/data/jmcp/JMCPMaga_363-380.pdf
8. Hartley H, Coleman CL. News media coverage of direct-to-consumer pharmaceutical advertising: implications for countervailing powers theory. Health (London). 2008;12(1):107-32.

9. Klonoff DC, Buse JB, Nielsen LL, Guan X, Bowlus CL, Holcombe JH, et al. Exenatide effects on diabetes, obesity, cardiovascular risk factors and hepatic biomarkers in patients with type 2 diabetes treated for at least 3 years. Curr Med Res Opin. 2008;24(1):275-86.

10. Singh-Franco D, Robles G, Gazze D. Pramlintide acetate injection for the treatment of type 1 and type 2 diabetes mellitus. Clin Ther. 2007;29(4):535-62.

11. Wysham C, Lush C, Zhang B, Maier H, Wilhelm K. Effect of pramlintide as an adjunct to basal insulin on markers of cardiovascular risk in patients with type 2 diabetes. Curr Med Res Opin. 2008;24(1):79-85.

12. Nauck MA, Meininger G, Sheng D, Terranella L, Stein PP. Efficacy and safety of the dipeptidyl peptidase-4 inhibitor, sitagliptin, compared with the sulfonylurea, glipizide, in patients with type 2 diabetes inadequately controlled on metformin alone: a randomized, double-blind, non-inferiority trial. Diabetes Obes Metab. 2007;9(2):194-205.

13. Feldstein AC, Nichols GA, Smith DH, Rosales AG, Perrin N. Weight change and glycemic control after diagnosis of type 2 diabetes. J Gen Intern Med. 2008; 23(9):1339-45.

14. Nathan DM. Finding new treatments for diabetes--how many, how fast... how good? N Engl J Med. 2007;356(5):437-40.

15. Richter B, Bandeira-Echtler E, Bergerhoff K, Lerch CL. Dipeptidyl peptidase-4 (DPP-4) inhibitors for type 2 diabetes mellitus. Cochrane Database Syst Rev. 2008(2):CD006739.

16. U.S. Food and Drug Administration. Information for healthcare professionals: exenatide (marketed as Byetta). Updated August 18, 2008. Available at: www.fda.gov/CDER/drug/InfoSheets/HCP/exenatide2008HCP.htm Accessed August 22, 2008.

17. U.S. Food and Drug Administration. Guidance for industry - diabetes mellitus: developing drugs and therapeutic biologics for treatment and prevention. February 2008. Available at: www.fda.gov/cder/guidance/7630dft. htm Accessed August 22, 2008.

18. Nathan DM, Buse JB, Davidson MB, Ferrannini E, Holman RR, Sherwin R Zinman B. Management of hyperglycemia in type 2 diabetes: a consensus algorithm for the initiation and adjustment of therapy. Diabetes Care. 2008;31(1):173-75. Available at: www.care.diabetesjournals.org/cgi/ reprint/31/1/173 Accessed August 20, 2008. 